that probe them, and the computation electromagnetics algorithms that model them. SPPs are composite excitations produced by the interaction of light with collective surface electron modes, known as surface plasmons, that exist at the interface between a metal and a dielectric. Many methods for coupling laser light into SPP modes have been demonstrated, using a variety of structures including prisms, gratings and nanoscale defects (such as surface protrusions or holes). But what has been missing is a means to ensure that the SPPs generated travel in one desired direction only, ideally from a nanoscale launching point.

The concept demonstrated by López-Tejeira for unidirectional SPP propagation is beautifully simple. It builds on a number of recent breakthroughs in the ability to excite and control the direction of their propagation ${ }^{5-9}$, yet relies on a number of subtleties. In essence, SPPs are launched on one side of a thin free-standing gold film by illuminating a subwavelength slit from the other side. Unidirectional propagation away from the slit is ensured by creating a regular pattern of small grooves to one side of the slit, which acts as a Bragg reflector for SPPs propagating towards it. Careful choice of the distance between the slit and the groove array allows the reflected SPPs to constructively interfere with the SPPs travelling in the opposite direction - the direction the authors wish them to travel.

The authors demonstrate this directed propagation at wavelengths of both $800 \mathrm{~nm}$ and in the telecommunication window around $1.5 \mu \mathrm{m}$, using near-field optical microscopy to directly image the near field of these propagating two-dimensional waves (see Fig. 1a). Using a variety of samples with different distances between the slit and the reflector array, both constructive and destructive interference in the desired propagation direction is demonstrated, in good agreement with theory. That it all works so well is a result of the fact that the reflective characteristics are determined only by the spacing of the grooves in the array, and are surprisingly insensitive to the precise shape of individual grooves, or even their number. This enables the authors to extend their approach to the construction of lensing elements made from an array of curved grooves that focus SPPs to a point (see Fig. 1b).

Could these slit couplers serve as interfaces between the outside world and nanoscale photonic waveguides, routing light from the excitation region to subdiffraction-limit spots? At least two different aspects of this scheme demand further investigation before this question can be answered. Firstly, the excited SPPs have to be channelled from the continuous film into laterally confined plasmon waveguides such as metallic slabs and nanowires, which should be relatively straightforward. The more important question is the total efficiency of the coupling, which the authors point out is a complicated function of the beam and slit profile, film thickness, and the excitation wavelength. For coupling schemes that use contra-directional (and therefore also unidirectional) evanescent power transfer between tapered optical fibres and

\title{
MAGNETIC RESONANCE IMAGING
}

\section{Take a close look}

Paul Lauterbur died on 27 March 2007, at the age of 77 . He will be remembered as one of the inventors of magnetic resonance imaging (MRI), a technique that revolutionized medical diagnosis. Lauterbur's now classic paper (Nature 242, 190-191; 1973) was rejected initially, something that he felt was a mistake, "not because I foresaw all of the medical applications that would follow, but because of the physical uniqueness of the concept", as he said in his 2003 Nobel Lecture.

Lauterbur's concept of 'image formation by induced local interaction' - which he proposed be known as 'zeugmatography', although the name didn't stick - uses the combination of two magnetic fields to find the location of spins: one field induces an interaction
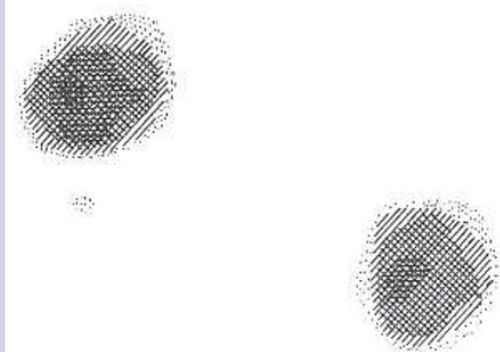

(that is, radio waves drive NMR transitions), whereas the other restricts this interaction to certain spatial regions. In this way, the image resolution is not limited by the wavelength of the radiation involved, as it is in conventional optics, but by the ability to disperse the interaction spatially using the second field.

This is key for MRI of humans. The radiofrequency fields used in a typical clinical MRI scanner have wavelengths of the order of a metre - wavelengths that ordinarily are "not sufficient even for imaging elephants", as Richard Ernst remarks in his textbook. But in his first demonstration of his zeugmatographic approach, Lauterbur produced resolved images of two water-filled capillaries, each with an inner diameter of only $1 \mathrm{~mm}$ (pictured, left). Over the years, the resolution of MRI experiments has been pushed further, down to a couple of micrometres. But this is still a long way from direct imaging of molecular structure - one of the visions of Peter Mansfield, who shared the 2003 Nobel Prize in Physiology or Medicine with Lauterbur.

The nanometre scale does, however, come within reach when MRI is combined with scanning probe microscopy, as John Mamin and colleagues now report

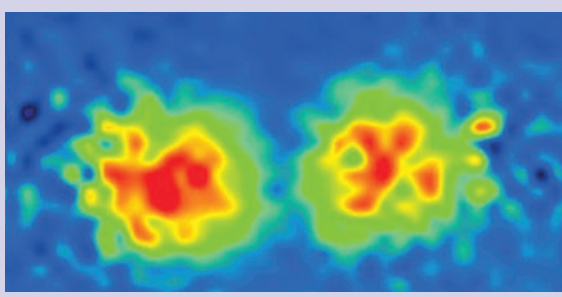

(Nature Nanotech. 2, 301-306; 2007). In magnetic resonance force microscopy (MRFM), a sharp tip that creates a localized magnetic field gradient is scanned across an object. In the experiment conducted by Mamin et al. the sample sits on a cantilever that has vibration characteristics that change whenever the sample is brought close to the tip. MRFM has been demonstrated before, but these authors have improved its resolving power considerably, not least by fabricating very sharp and highly magnetized tips, which guarantees both high spatial resolution and a strong signal. This and other improvements have paved the way to a record resolution of $90 \mathrm{~nm}$, or an imaging volume of less than an attolitre (the objects in the coloured images above are each less than $300 \mathrm{~nm}$ ). Mamin et al. estimate that only a few thousand nuclear spins contributed to the signals they detected. Andreas Trabesinger 